\title{
Strategic Investment in Laboratory Analysis of Planetary Materials as Ground Truth for Solar System Exploration
}

Rhonda Stroud (Naval Research Laboratory, rhonda.stroud@nrl.navy.mil, 202-404-4143)

Jessica Barnes (University of Arizona) Larry Nittler (Carnegie Institution for Science) Juliane Gross (Rutgers University) Jemma Davidson (Arizona State University) Catherine Corrigan (Smithsonian Museum of Natural History)

Hope Ishii (University of Hawaii) Jamie Elsila Cook (NASA Goddard Space Flight Center) Justin Filiberto (Lunar and Planetary Institute) Samuel Lawrence (NASA Johnson Space Center) Michael Zolensky (NASA Johnson Space Center) Devin Schrader (Arizona State University)

Barbara Cohen (NASA Goddard Space Flight Center) Kevin McKeegan (University of California, Los Angeles) and CAPTEM. 
Introduction: Laboratory analysis of planetary materials provides a wealth of information critical to solving fundamental questions in planetary science that cannot be replicated through remote or in situ analyses alone. Our ground-based collections include samples of the Moon, Mars, the Solar corona, and dozens of asteroids and comets. This vastly exceeds the number of targets of past, and future fly-by, orbiter, or lander missions in the next decade. Similarly, the combined analytical power of the laboratory instrumentation available now vastly exceeds that of the weight and power-constrained spacecraft and rover instrumentation that has already and can be flown this decade. If we are able to put new human footprints on the Moon and Mars within the next few years, as it is now hoped, those astronauts will go propelled in part by the information gleaned by thousands of researchers who have already held the Moon and Mars in their hands. The work of laboratory researchers has revealed and continues to uncover the imprints of solar system processes that provide ground truth for fly-by, orbital, lander and rover missions. The researchers who perform these studies, the advanced analytical equipment they wield, and the samples they investigate are key resources that need on-going investment to evolve with the continuously advancing goals of the planetary science and exploration communities. We advocate for a robust allocation of resources needed to curate the existing samples, conduct targeted expansion of the collections, train the next generation of planetary materials analysts, and advance the state-of-the-art in sample analysis methods.

The decisions about how to most effectively invest resources for laboratory analysis must be made in the context of the overarching goals of planetary science, the state of the current collections, future curation needs, emerging instrumental methods, and broad engagement of a demographically and disciplinarily diverse workforce. The specific focus of this whitepaper is to communicate the scientific value of the existing collections to the overarching goals of the planetary science community, and to highlight the highest priority and most timely areas for expanding the collections and analytical instrumentation. Related whitepapers address complementary topics, including: advanced curation [1], experimental laboratory sample analyses [2] new sample return targets, such as unexplored Lunar regions, Mars [3], Mercury [4], comet surface [5], cryogenic comet sample return [6], gas/ volatile return [7], Ceres, Vesta and other small bodies [8], Earth-based extraterrestrial sample recovery [9] and workforce diversity.

Status of Sample Collections as Resources for Planetary Science: At present, there are six distinct major sample collections curated by NASA at Johnson Space Center: Apollo lunar samples, Antarctic meteorites (ANSMET), Cosmic Dust, STARDUST cometary and interstellar dust, Genesis solar wind samples, and Hayabusa Itokawa asteroid regolith grains. Over the next decade, the collections will expand to include OSIRIS-REx and Hayabusa2 samples of asteroids Bennu and Ryugu, respectively, new lunar samples, and possibly the first samples brought back from the surface of Mars. A small selection of the many key planetary science questions that can be addressed with new and ongoing studies of these samples includes: What is the inventory and evolution of organic molecules in the solar system? What were the origins of water on Earth? What is the composition of the Sun? What processes are involved in planetary accretion and differentiation? What are the effects of space weathering on airless bodies under differing radiation, impact and thermal conditions? What are the process of nebular transport? and What is the composition of contemporary interstellar dust delivered to the solar system? 
Apollo Lunar Sample Collection: Our understanding of the Moon and its early history have been revolutionized through the study of returned Apollo samples. The lunar crust records and preserves an archive of planetary history. Over the last half century, the returned Apollo samples have given us a glimpse into the early history and broad-scale petrogenesis of the Earth-Moon system, planetary formation, evolutionary processes, and our solar system history; the returned samples from the lunar crust also provided us with an understanding of the history of the solar system climate through the interaction with solar wind, galactic X-rays, and early solar system materials that impacted the lunar surface (and most likely Earth). Today, models of lunar crust formation serve as a benchmark for understanding crustal formation and evolution throughout the entire solar system. Many of these discoveries have been made only in the last decade due to the invention of new and more advanced and sophisticated technologies and because the Apollo samples have been maintained in a pristine manner and remain available to the scientific community. With the continued study of the Apollo collection and new samples returned from unexplored terrain, future generations of scientists will continue to extract surprises and make profound discoveries, and the returned samples (old and new) will continue to hold greater and increasingly novel value beyond the initial studies half a century ago. The long-lasting legacy of sample return from the Moon is a key component of a holistic way of studying our solar system.

Antarctic Meteorite Collection: Since 1976, the understanding of our solar system has been greatly enhanced by the 23,000+ meteorite samples recovered by the U.S. Antarctic Meteorite program (field collection by the Antarctic Search for Meteorites - ANSMET - and classification/curation by Johnson Space Center and the Smithsonian). With these samples, which have been loaned to hundreds of researchers worldwide, we have found asteroids containing the most primitive materials in our solar system as well as those that have been variably geologically processed. We have pieces of asteroids that formed with ice and were subsequently aqueously altered, and those that contain organic compounds such as amino acids and sugars that could possibly represent the precursor components of life on Earth. We have pieces of asteroids that formed with no exposure to water, those that inform studies of impact processes, melting (with and without available oxygen), samples from unknown locations on the Moon, samples of Mars (including the only hand-sample evidence of liquid water on its surface), and presolar grains actual grains from solar systems that existed before our own. These meteorites, many of types not known before ANSMET, have forced us to ask new questions about conditions and processes that take place during planetary differentiation and core formation, and so much more. Using these samples, including the first recognized lunar meteorite (a recognition made possible by comparison to Apollo samples), the first recognized martian meteorite (discovered by comparison of trapped gases within the rock to Viking's compositional measurements of Mars' atmosphere) and the HED meteorites available to compare with 4 Vesta, we have also expanded our knowledge of the solar system with complementary mission data, and in some cases, (e.g. OSIRIS-REx and Psyche), used meteorites to inform mission planning.

Cosmic Dust Collection: Many thousand tons of cosmic dust from all dust-producing bodies, including asteroids and comets, settle through Earth's atmosphere each year. Stratospheric and ground-based collections of this cosmic dust provide invaluable context for interpreting comet and asteroid returned samples and motivating future sample return missions [9]. Since 1981, NASA has collected cosmic dust with stratospheric aircraft-based collectors, and in the past decade, 
specific comets and asteroid families were targeted in timed collection flights. More recently, alternative collection methods are being explored, including high volume air sampling in clean locations, balloon flights, and new collection substrates. Cosmic dust is curated in a dedicated ultra-clean laboratory at NASA's Johnson Space Center using highly specialized methods to handle particles a few microns in size [10]. Among the wide range of asteroidal-type cosmic dust particles, just a handful have been directly linked to known meteorite classes, and therefore many represent asteroid parent bodies not yet found in meteorite collections. Cometary-type particles contain the highest measured abundances of preserved presolar grains, identified by isotope anomaly signatures of their origins in environments around other stars. These cometary particles are the most primitive material available for study on Earth, providing insight into the starting materials and earliest stages of planetary body formation prior to the differentiation experienced by larger bodies. Prior to NASA's Stardust mission (below), the only comet samples for laboratory study were cosmic dust, and the resulting understanding of the diversity of extraterrestrial bodies and of cometary-type dust contributed enormously to Stardust mission science goals, as well as informing comet fly-bys and the ROSETTA mission.

Genesis Solar Wind Collection: NASA's Genesis Discovery mission returned solar wind atoms implanted into a range of high-purity materials (including silicon, silicon carbide, sapphire, diamond, germanium, gold, and aluminum) in 2004. Despite the unfortunate crash of the sample return canister upon return to Earth, which caused most of the collectors to shatter and introduced terrestrial contamination, most of the highest-level original science goals of the mission were accomplished. In particular, the determination of the $\mathrm{O}$ and $\mathrm{N}$ isotopic compositions of the Sun, was of high importance to many problems of both cosmochemistry and planetary science. Since then, Burnett et al. [11] have identified 12 additional compelling science objectives still to be addressed with the Genesis samples, ranging from constraint of solar wind acceleration processes through determination of the abundances of elements with different ionization potentials, to better understanding of variations among solar system materials through determination of the heavy elemental isotopic compositions, to constraining the origin and evolution of lunar volatiles through $\mathrm{C}$ isotope measurements. Much of the science accomplished so far with Genesis samples required development of analytical methods and techniques that did not exist before the mission was launched and further technique development will be required to meet the new objectives. The Genesis curatorial facility at JSC includes a dedicated Class 10 cleanroom laboratory that provides secure storage of the $>10,000$ collector fragments that comprise the collection as well as capabilities and instrumentation for sample preparation and characterization (e.g., ellipsometer, FTIR, automated microscope).

Stardust Comet and Interstellar Dust Grains: NASA's STARDUST Discovery mission provided two unprecedented and unmatched resources for the planetary science community: comet dust samples from a known comet parent body (81P/Wild) [12], and dust from the contemporary interstellar dust stream [13]. With these samples, returned in 2006, researchers address basic questions about the composition of the earliest solid building blocks on the solar system, and what dust components are available in the interstellar medium today for incorporation into new solar systems. Stardust cometary sample analyses continue to expand our understanding of the diversity of materials at the earliest stages of planetesimal formation, ranging from amino acids such as glycine, to refractory organic nanoglobules, to refractory silicates and calcium, aluminum-rich 
inclusions. Results from Stardust samples point to wide-scale transport and mixing of dust reservoirs between comet and asteroid forming regions, and that comets represent much more than cold storage of pristine dust from interstellar sources. With no selected comet sample return mission in the current cadence, the 81P/Wild samples will remain our only comet dust sample of known origin until at least the late 2030's. The analyses of the Stardust interstellar samples to date provides high confidence that the collection preserves interstellar dust, but definitive evidence in the form of an oxygen isotopic composition outside the range of Solar System values for these grains has yet to come. Future improvements in the technology for isolating the grains captured in aerogel and/or obtaining the oxygen composition in situ are necessary to fully achieve the potential of this collection.

Hayabusa Itokawa Regolith Grains: Regolith grain samples of S-class, near-Earth asteroid 25142 Itokawa were returned to Earth by the Hayabusa spacecraft on June 14, 2010. The JAXA Hayabusa curation laboratory in Sagamihara, Japan houses $90 \%$ of the samples. The remaining $10 \%$ are in a small dedicated lab at NASA's Johnson Space Center Curation Facility. In both curation labs the samples are maintained in curation grade nitrogen gas-filled glass and steel cabinets or in a vacuum. This level of care is necessary to maintain the pristine nature of the Sclass asteroid samples whose collection will not be repeated for decades. The Itokawa samples have been isolated from Earth's atmosphere to a degree not duplicated for meteorites. Such care is required as atmospheric sensitive minerals and organics are present which hold a unique record of the early history of the solar nebula inside of the snow line, and the origin history of S-class asteroids. Detailed sample analyses demonstrated that Itokawa consists of thermallymetamorphosed ordinary chondrites, verification that ordinary chondrites derive from S-class asteroids [14]. The detailed thermal and shock history of the samples is now being teased out in ground-based labs performing measurements not possible by spacecraft. Such information is only available from carefully collected and curated samples, and ground-based measurements with state of the art facilities. That these particles are directly available to US researchers from the JSC curation facility will greatly facilitate the NASA-funded communities' access to the samples in a timely manner.

In-flight C-type Asteroid Sample Return Missions: With two sample-return missions currently in-flight - JAXA's Hayabusa2 mission and NASA's Origins, Spectral Interpretation, Resource Identification, Security, Regolith Explorer (OSIRIS-REx) New Frontiers mission - we are in a golden age of extraterrestrial sample return. The Hayabusa2 and OSIRIS-REx missions to nearEarth asteroids share similar goals of understanding the origins of primitive, hydrated, organicrich asteroids. Hayabusa2 was launched on December 3, 2014 and rendezvoused with near-Earth C-class asteroid 162173 Ryugu on June 27, 2018. After a detailed survey of the surface of Ryugu, Hayabusa2 made two successful touchdowns on the surface, collecting two separate samples [15]. The spacecraft departed Ryugu in November 2019 and is expected to return samples to Earth on December 6, 2020. The OSIRIS-REx spacecraft was launched on September 8, 2016 and rendezvoused with near-Earth B-class asteroid 101955 Bennu on December 3, 2018. The first Touch-And-Go regolith sampling attempt is scheduled for October 2020. Samples are expected to arrive on Earth in September 2023. The Hayabusa2 and OSIRIS-REx missions plan to return 0.1 to $10 \mathrm{~g}$ and 0.06 to $2 \mathrm{~kg}$ asteroid regolith material, respectively [16, 17]. Under an agreement between JAXA and NASA, $10 \%$ of the Ryugu material will be shared with NASA and $0.5 \%$ of 
Bennu materials returned by OSIRIS-REx will be sent to JAXA. The Hayabusa2 sample storage and handling facility and the OSIRIS-REx clean sample curation facility are under construction at NASA JSC [10]. These new collections will vastly improve our ability to connect remote sensing observation of primitive carbon-rich asteroids to their structural and mineralogical properties, and accretion and processing histories.

Instrumentation Development / Facility Support: A guiding principle of laboratory cosmochemistry is that the scientific payoff of sample collections grows over time, hand-in-hand with the advancement of analytical instrumentation [18]. This is true in practice only if there is systematic investment in sample curation, instrument development, analytical facilities, and development of the experienced workforce. In the case of returned lunar samples, the principle has been beautifully illustrated through new analysis of long-curated Apollo samples with state-of-art x-ray and electron microscope technology through the Apollo Next Generation Sample Analysis (ANGSA) program. The samples were held unopened, for nearly 50 years, in anticipation of the eventual availability of appropriate curation and analysis tools for investigation of incorporated volatiles. As a first step to the sample process for allocation, the entire sealed core sample was mapped in three-dimensions at the University of Texas High-Resolution X-ray Computed Tomography Facility. With this tomographic data (Fig. 1A)-D)), the stratigraphy and mineralogy has been revealed in scales down to $<10$ microns, which will enable allocation of targeted component materials to each researcher in the consortium for measurements with a wide variety of specialized instrumentation for volatile, isotopic and mineralogical analyses at scales down to the individual atoms (Fig. 1 E) \& F)), to better reveal the history of the lunar regolith. The next step is coordinated analysis in more than a dozen state-of-the art laboratories that will provide the most complete depth profile of the Lunar regolith. It would have been impossible to package a CT scanner, scanning electron microscope, ion microprobe and noble gas mass spectrometer to collectively or even individually fit in the Apollo 17 payload that returned the ANGSA samples, and even if had been possible, the limited data storage and processing capabilities of the early 1970s alone would have made such studies impossible. It is through the leveraging of 40+ years of computer and instrumentation advances, and persistent, careful curation that such powerful studies are possible. While the community could not have anticipated that after Apollo 17 we would not return additional samples from the Moon until at least 2024, it is a stark reminder that the sample collections are a near-term investment in today's dollars with untold compound returns over multiple generations.

The full case for investment in analytical facilities for returned sample analysis was laid out in the 2018 National Academies report, "Strategic Investments in Instrumentation and Facilities for Extraterrestrial Sample Curation and Analysis.” [18] Key findings of this document include the need to support facilities in both individual PI and user facility formats, and to share costs across funding agencies, while investing strongly through NASA-funded instrumentation and analysis programs so that the specific needs of the planetary science community are realized. Key laboratory analysis technologies that will require significant investment in the coming decade, and can leverage advances in the larger scientific community, include sample handling and measurements under cryogenic conditions for handling of easily damaged organics and volatile samples, including ices and entrained gases. 

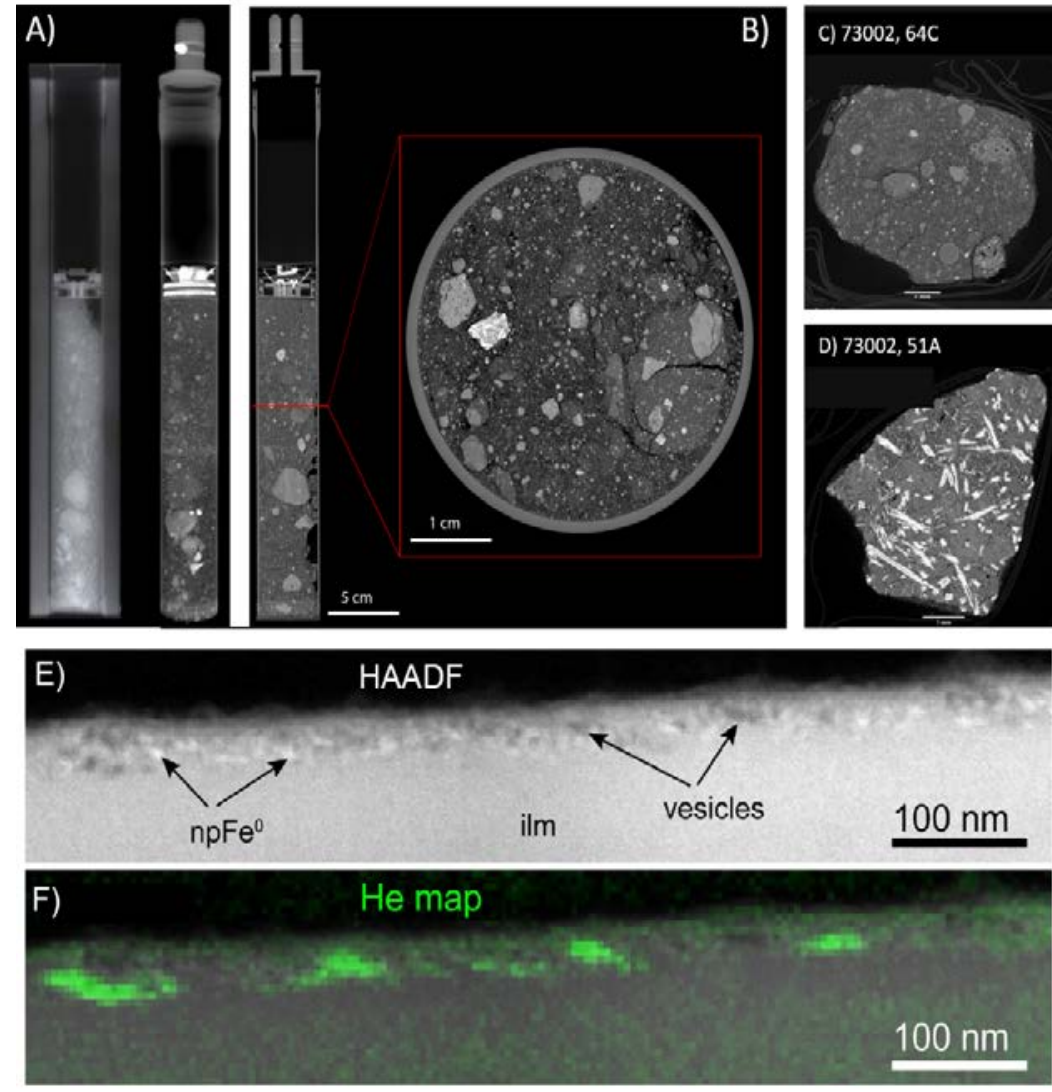

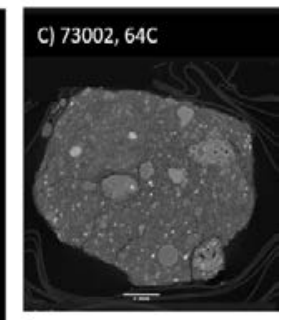

D) $73002,51 \mathrm{~A}$

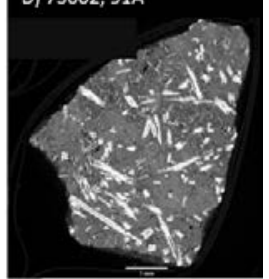

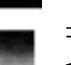

https://ui.adsabs.harvard.edu/\#abs /2020LPI....51.3023Z/abstract. [1]. E) and F) Dark field STEM image and STEM -EELS map of a lunar soil ilmenite grain, showing the formation of nanophase $\mathrm{Fe}\left(\mathrm{npFe}^{0}\right)$ and concentration of solar wind helium in vesicles. After DOI: 10.1016/j.gca.2017.12.023.

Future Sample Return Missions, and Challenges: Exploration of more regions of the solar system through sample return missions targeting a variety of bodies have been, and will continue to be, proposed to NASA opportunities and/or supported by international partners. Currently planned or programmed NASA missions include sample return from the Moon (Artemis) and Mars, while JAXA's Martian Moons eXploration (MMX) mission aims to return samples from Phobos. Proposed mission concepts envision sample return from the plumes of Enceladus and the nucleus of a comet, among other targets. Such missions are the culmination of a decades-long pipeline progressing from fly-bys to orbiters, landers, rovers, and finally, sample return. NASA's recent Announcements of Opportunity for Discovery and New Frontiers Programs specify that returned samples will be curated at JSC, with "actual costs for all aspects of curation, from planning through distribution and storage, including all required laboratory construction or modification ... borne by the mission from inception to two years following sample return.”

Future returned samples are likely to include not only rocks and solid refractory materials, but also volatiles, ices, liquids, and labile materials that would enable key scientific investigations, but which would also require specialized sampling, curation, and manipulation capabilities to minimize losses and alteration and enable long-term preservation [7, 8]. Dedicated curation laboratories would need to be designed and constructed, with attention paid to the types of samples to be collected by each mission. New technologies (e.g., for cryogenic storage, manipulation, and subsampling) would be required [10]. 
A high priority for lunar exploration is the collection of new samples and their return to Earth for comprehensive analysis. Sample return from a range of locations, including South PoleAitken, would yield important advances in planetary science [19, 20]. NASA's Project Artemis is expected to enable human expeditions beyond low-Earth orbit for the first time in five decades, with the goal of establishing the Artemis Base Camp field station at the lunar South Pole by 2028. Geologic sample return was levied as a requirement on vendors proposing human lunar landers, with the expectation that at least $23 \mathrm{~kg}$ of rock samples will be returned on each Artemis mission. The polar landing sites offer the opportunity to return volatile-enriched samples from the Moon for the first time from permanently shadowed regions found at the lunar poles.

Future samples returned from Mars would necessitate a combined effort to preserve sample integrity while also meeting planetary protection requirements [3]. Mars samples are expected to be screened for evidence of possible life and biohazards in a dedicated sample receiving facility (SRF). Once the samples can be shown to contain neither life nor hazard, or if portions can be effectively sterilized, they would be released from the SRF for curation at NASA JSC and distribution to the community. Specialized requirements may include robotic sample handling, preservation of temperature- and humidity-sensitive materials, and of atmospheric samples.

International cooperation with JAXA's MMX mission could bring samples from the martian moon Phobos to Earth in the next decade. As with Hayabusa and Hayabusa2, international collaboration could result in samples to be curated at JSC. These samples are not subject to the same level of planetary protection constraints as Mars samples, while still providing unparalleled insight into the history of Mars and its moons.

The success and scientific impact of these sample return missions depend not only on appropriate investment in the development, construction, and maintenance of cutting-edge facilities, but also on investment in developing a new, diverse generation of sample scientists. Broad engagement of young researchers across discipline and demographic boundaries is needed to invent, test, and carry out the necessary sample processing and curation procedures, and to share knowledge of sample analyses with the larger planetary science community. Therefore, continued support in the next decade is essential for both the continued hiring and training of such personnel to advance laboratory science of planetary materials, to maintain current and future collections of returned samples, and in the development of necessary advanced processing and preparation techniques to prepare for new types of returned solar system materials.

References: 1. McCubbin et al., Adv. Curation of Astromater. for Planet. Sci. (submitted whitepaper). 2. Fayolle et al. Critical. Lab. Studies...(submitted whitepaper); Horányi et al. Interplanet. and interstell. dust...(submitted whitepaper). 3. McSween et al., Why Mars Sample Return...(submitted whitepaper). 4. Vander Kaaden et al.., Mercury Sample Return...(submitted whitepaper). 5. Nakamura et al.., Comet Surface Sample Return. (submitted whitepaper). 6. Westphal et al. Cryogenic Comet Sample Return. (submitted whitepaper). 7. Milam et al., Volatile Sample Return...(submitted whitepaper). 8. Jacobson et al. Small Body Sample Return (submitted whitepaper). 9. Ishii et al. Terrestrial Collection of extraterr. mater.. (submitted white paper). 10. 10.1007/s11214-019-0615-9. 11. 10.1111/maps.13266. 12. 10.1126/science.1135840.

13. $10.1126 /$ science.1252496. 14. $10.1126 /$ science.1207758. 15. $10.1126 /$ science.aaz6306.

16. $10.1007 / \mathrm{s} 11214-017-0405-1.17 .10 .1007 / \mathrm{s} 11214-017-0377-1$ 18. 10.17226/25312.

19. 10.1029/2018EA000490. 20. https://www.hou.usra.edu/meetings/V2050/pdf/8142.pdf 